INPLASY

PROTOCOL

To cite: Huang et al. Outcomes of septic shock adult patients receiving extracorporeal membrane oxygenation: A pooled experience of 1,895 cases. Inplasy protocol 202070040. doi:

10.37766/inplasy2020.7.0040

Received: 11 July 2020

Published: 11 July 2020

Corresponding author: Hui-Bin Huang

hhba02922@btch.edu.cn

Author Affiliation:

Beijing Tsinghua Changgung Hospital

Support: This work was supported by NSF.

Review Stage at time of this submission: Preliminary searches.

Conflicts of interest:

The authors declare that they have no competing interests.

\section{Outcomes of septic shock adult patients receiving extracorporeal membrane oxygenation: A pooled experience of 1,895 cases}

\author{
Huang, HB1; Zhou, H²; Zhang, XJ3; Xu, Y4; Du, B5.
}

Review question / Objective: We aim to conduct a systematic review and meta-analysis of the available data to assess the clinical outcomes of SS adults receiving extracorporeal membrane oxygenation (ECMO), and to determine the factors associated with prognosis.

Condition being studied: Our team members come from a tertiary hospital in China and all the members have extensive experience in treating septic shock with ECMO. Moreover, our team members have published several meta-analyses, which can guarantee the completion of the current study.

INPLASY registration number: This protocol was registered with the International Platform of Registered Systematic Review and Meta-Analysis Protocols (INPLASY) on 11 July 2020 and was last updated on 11 July 2020 (registration number INPLASY202070040).

\title{
INTRODUCTION
}

Review question / Objective: We aim to conduct a systematic review and metaanalysis of the available data to assess the clinical outcomes of SS adults receiving extracorporeal membrane oxygenation (ECMO), and to determine the factors associated with prognosis.
Rationale: Septic shock (SS) is still a leading cause of death in critically ill adults. ECMO has shown to improve survival of patients with severe respiratory failure or cardiac shock. However, for adults, the effectiveness of ECMO on SS remains controversial and no guideline or metaanalysis available. Recently, with ECMO 
technological development, use of ECMO in adult has greatly increased. However, most studies are observational, and the results are often contradictory.

Condition being studied: Our team members come from a tertiary hospital in China and all the members have extensive experience in treating septic shock with ECMO. Moreover, our team members have published several meta-analyses, which can guarantee the completion of the current study.

\section{METHODS}

Search strategy: Two investigators systematic searched potentially relevant studies in PubMed, EMBASE, and Cochrane database. The search terms were "extracorporeal membrane oxygenation", "extracorporeal life support", "ECLS", "ECMO" and "septic shock". No language limitation was imposed.

Participant or population: Studies were considered for inclusion if they evaluated septic shock adults receiving ECMO support.

Intervention: Adult patients receiving ECMO during their stay in ICU.

\section{Comparator: Without limitation.}

Study designs to be included: Studies with all typr of designs are considered for inclusion if they evaluated septic shock adults receiving ECMO support.

Eligibility criteria: We included trials of adults ( $\geq 18$ yr or older) admitted to an ICU.

Information sources: The references in the included studies and personal files were also searched. In addition, we will request advice from experts in the field; search associated articles from critical care, surgical, infection meetings; and contacted the authors of included trials, if need.

Main outcome(s): The primary outcome: short-term mortality (defined as ICU or hospital mortality or mortality within a 90 - day follow-up after admission, with the longest observation period preferred). Secondary outcomes: ECO weaning successfully, duration of mechanical ventilation, length of stay (LOS) in ICU or hospital, long-term mortality (defined as mortality between hospital discharge and at least one year follow up thereafter) and adverse events (AEs).

Quality assessment / Risk of bias analysis: The Newcastle-Ottawa scale (NOS) for observational studies will be used to assess the quality of the included studies. The NOS statement was judged on three broad perspectives (selection, comparability, and outcome) consisting of eight items. The qualities of RCTs were assessed using the risk of bias tool recommended by the Cochrane Collaboration. We assigned a value of high, unclear, or low to several parameters: 1) random sequence generation; 2) allocation concealment; 3) blinding of participants and personnel; 4) blinding of outcome assessment; 5) incomplete outcome data; 6) selective reporting; and/or 7) other bias.

Strategy of data synthesis: The differences between the two groups were calculated as the relative risk (RR) / mean difference (MD) and $95 \%$ confidence interval (CI) for according outcomes. Heterogeneity was assessed by the Cochran $Q$ statistic and the I2statistic. A $P$ value $\leq 0.10$ together with an 12 value $\geq 50 \%$ indicates significant heterogeneity. 12 values $\leq \mathbf{5 0} \%$ represented acceptable between-study heterogeneity, and the fixed-effects model was selected. Otherwise, the random-effects model was selected.

\section{Subgroup analysis: None.}

Sensibility analysis: We aim to conduct analyses by pooled studies with the following: (1) hospital mortality; (2) 30-day mortality; (2) 60-day mortality; (3) 90-day mortality; (5) mortality in patients receiving venovenous (VV) mode; (6) mortality in patients receiving VA mode; (7) mortality in patients with VA mode and cardiac dysfunction (i.e., decreased ejection fraction, EF). 
Language: No language limitation was imposed.

Country(ies) involved: China.

Keywords: extracorporeal membrane oxygenation, septic shock, meta-analysis, mortality.

Contributions of each author:

Author 1 - Hui-Bin Huang - H-BH will be responsible for conception of the study, searching the scientific literature and drafting the manuscript.

Author 2 - Hua Zhou - HZ will be responsible for conception, design and data interpretation.

Author 3 - Xiang-Jun Zhang - X-JZ will help to collect the data and performed statistical analyses.

Author 4 - Yuan Xu - YX will be responsible for conception, design, data interpretation, manuscript revision for critical intellectual content, and supervision of the study.

Author 5 - Bin Du - BD will be responsible for conception, design, data interpretation, manuscript revision for critical intellectual content, and supervision of the study. 